\title{
Low-cost Laboratory Stand for Turbidity Measurements
}

\author{
Lukasz Makowski ${ }^{1}$ \\ ${ }^{1}$ Faculty of Electrical Engineering, Warsaw University of Technology, \\ ul. Koszykowa 75, 00-662 Warszawa \\ lukasz.makowski@ee.pw.edu.pl
}

\begin{abstract}
Fresh water becomes scarcity not only worldwide but also in some developed countries. Therefore there is more and more attention paid to quality of this exceptional natural resource. Clarity or its inverse known as turbidity is key feature of water characteristic. Smaller, easier to use and more convenient device to measure this parameter could be constructed with significantly lowered costs thanks to improvements in electronic components. Research presented in this paper is aimed at constructing a laboratory stand with which a progress could be made in development of such device. Construction of optical system with limited set of items is discussed as well as design and assembly of opto-electronic transducer.
\end{abstract}

Index Terms-Optoelectronic devices, laser applications, optical sensors, pollution measurement, environmental monitoring.

\section{INTRODUCTION}

Even though recent century brought huge civilizational advancement and technical development, access to clear drinking water is still an issue. Surprisingly even in Europe countries like Spain and Poland have fresh water resources per capita similar to Egypt. It might be postulated that electronic measurement systems should become more common for better management of this precious resource.

There are many quality factors that determine if water is suitable for specific needs. Clean water requirements are established in legal documents created by international organisations like WHO or national bodies like EPA in USA. To EU countries applies "Drinking Water Directive" that enumerates checklist of parameters which have to be measured on regular basis and turbidity is one of them [1].

Light is an electromagnetic wave and it interacts with matter through which it is travelling. It may scatter on solid particles that are suspended in water. Presence of such particles is simple yet important indicator of water quality. These suspended solid particles when in higher concentrations cause haziness of water that is visible with naked eye. However, water expected for consumption should have turbidity so low that the haziness must not be observed without specific equipment.

Characteristic of scattering depends on particles size and wavelength of illuminating light [2]. Measured turbidity $T$ is

Manuscript received 24 February, 2015; accepted 14 September, 2015.

This research was funded by a statutory funding from the Faculty of Electrical Engineering at Warsaw University of Technology. This research was performed in cooperation with the Institution. proportional to inverse of wavelength $\lambda$ of light which is penetrating the sample. Smaller particles such as single atoms or molecules scatter only shorter wavelengths. It is assumed that in such case light is evenly spread in all directions. This theory is known as Rayleigh scattering and it also explains why sky has blue colour. Mie-Lorenz theory that was developed later explains thoroughly more general case in which particles have size similar to the wavelength of irradiating light [3].

Many designs of turbidity meters were proposed. Development nowadays is focused on light source, sensors and effect of angular aligning sensor with lightpath (i.e. angular functions of scattered light) [4], [5]. Introduction of laser as a light source improved measurements quality by terms of lowering the uncertainty [6]. Advancements in electronics and particularly the laser technology both enhances and simplifies multi-angular light scattering by providing measurements with reduced truncation angle [7]. Furthermore, there are in-situ, wireless systems that measure water parameters including turbidity in fresh water reservoirs [8], [9].

However, commercially available turbidimeters are still quite expensive instruments. Cost of devices must drop significantly to make turbidity measurements more accessible to society. Research presented in this article is an answer to such demand.

\section{OPTICAL SYSTEM ON BREADBOARD}

Optical part of stand construction consists of laser light that illuminates spectrophotometric cuvette which contains tested sample and which is surrounded by pinholes and plastic polarisers. All components were aligned on optical breadboard so that light is properly coupled into a fibre. Schematic diagram of the breadboard is shown on Fig. 1.

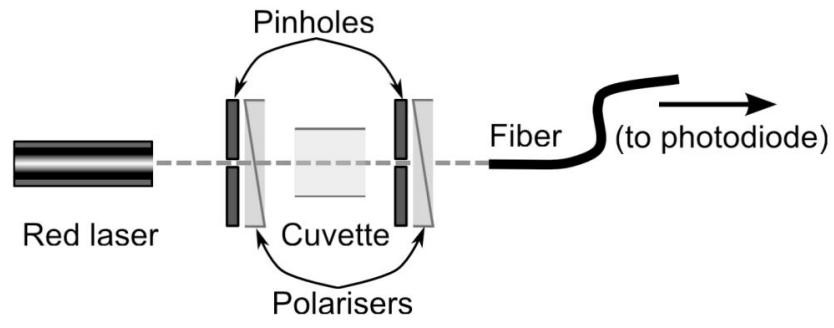

Fig. 1. Optical breadboard diagram.

An inexpensive class 3B red diode laser module ML-33S$660-80$ made by Semicon was used as a beam source. It 
provides light at $660 \mathrm{~nm}$ wavelength with power of $80 \mathrm{~mW}$. Laser collimation is possible thanks to one movable lens out of four that form this laser optics. Having no professional laser holder it was necessary to find another way to hold it tightly yet still maintain its positional control. This issue was solved with slightly modified laser mount that was originally designed to be an aim helper (a "finder") for astronomical telescopes. Laser tubular enclosure with diameter of $20 \mathrm{~mm}$ fits well in the holder and leaves some space $(1,5 \mathrm{~mm})$ for adjustment of its position. Laser holder can be rotated and moved in $\mathrm{Z}$ axis. It might be secured in one position with PF175 clamping fork made by Thorlabs. Final assembly of laser in mount is presented on Fig. 2.

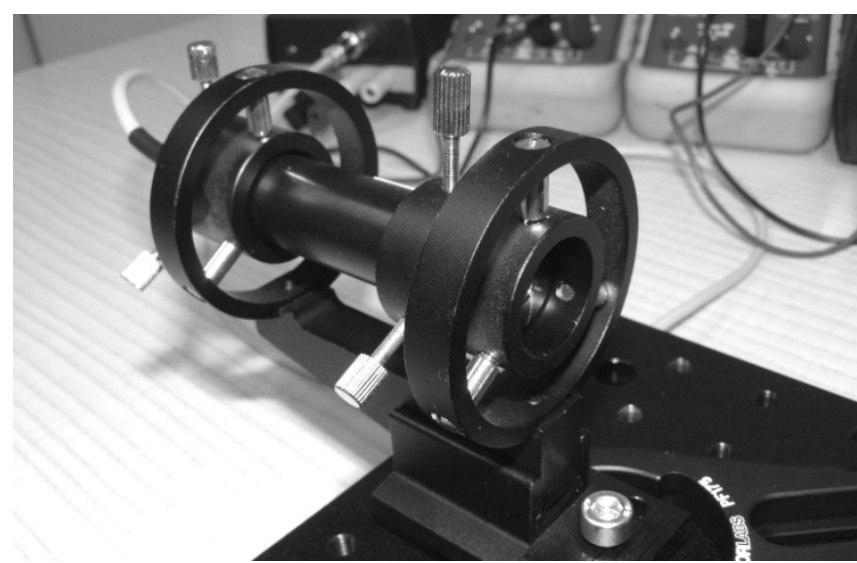

Fig. 2. Inexpensive laser mount used in astronomy adapted to optical breadboard. Thorlabs PF175 clamping fork holds it in place.

High quality spectrophotometric glass cuvette is used to hold a material under test. Its light path length equals 10 mm. Sides of the cuvette are matted to be semitransparent. A cap made of Teflon helps to keep the material clean from dust that could otherwise contaminate sample. Cuvette holder was constructed in the lab. Its first version was made of stiff foam and it also worked as early variant of pinholes mount.

Light beam goes through a round pinholes of $0,6 \mathrm{~mm}$ diameter that were crafted in the lab. These components minimize light artefacts and narrow the beam diameter. Former pinholes were made in several layers of polarizing foil epoxied onto aluminium sheet. Such pinhole was put into slice in the foam so that it was possible to move it in $\mathrm{YZ}$ plane and rotate along $\mathrm{X}$ axis. Once placed it remained in position quite reliably so this solution worked out surprisingly well. However, impossibility to move pinholes along $\mathrm{X}$ axis - to move it towards or further from cuvette were reason to try a different approach.

Current pinholes were drilled in steel sheet and then small pieces of polarizing foil were attached on both pinholes. Such package was epoxied to SM05PT threaded adapter which fits SMR05 holder. By rotating an adapter to which a pinhole with polariser is attached it is possible to attenuate light of the laser. Therefore in current system top power that leaves the pinhole might be set and so a maximum light that can enter the fibre as well. Adjusting this value provides some control over peak voltage provided to ADC in light-tovoltage converter circuit. SMR05 is placed on post with post holder so now it is possible to adjust pinholes positions in all planes and rotate it along $\mathrm{Z}$ axis and along beam axis.

As an early version of the cuvette-and-pinholes holder cut in foam became obsolete it was decided to construct new version just for the cuvette. Now it is made of bended steel stripe that was covered with heat shrink for glass safety and then screwed onto solid pedestal pillar. It might be moved in the breadboard plane. Moving along $\mathrm{Z}$ axis is not that important as cuvette height creates tolerance for elevation of other optical components. Final arrangement of optical components is shown on Fig. 3. Scattering of laser light might be observed in the cuvette that was holding some sample.

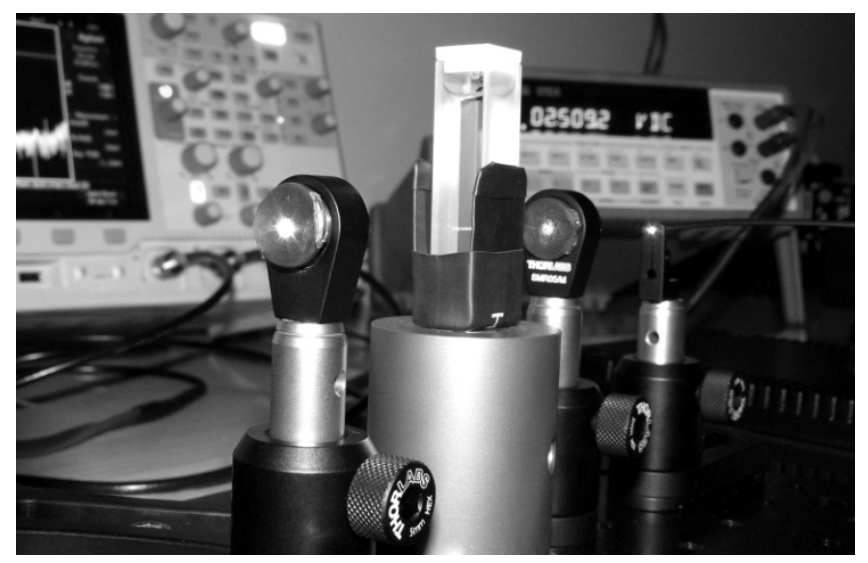

Fig. 3. Elements on optical breadboard. Laser (not shown) operates from the left. Then from left to right: polariser with pinhole on post, cuvette on post, second polariser with pinhole, fibre entry point.

Preliminary experiments with professional glass fibre (125 $\mathrm{m}$ of diameter) proved that without more advanced launch platform it is hardly possible to correctly couple the light into fibre. Perhaps it is possible with components that are currently available to author but so far there was no success. Therefore simpler alternative with plastic fibre was used and it proved to be low-cost yet reliable solution.

\section{CONSTRUCTION OF ElECTRONIC TRANSDUCER}

Fibre is used to transmit light to an optoelectronic transducer with which a measurement is performed. Main element of the transducer board is Texas Instruments OPT101 amplified photodiode. This chip should be considered inexpensive but effective solution in many designs. Its photodiode area is larger than $5,22 \mathrm{~mm}^{2}$ and it has responsivity higher than $0,45 \mathrm{~A} / \mathrm{W}$ at $660 \mathrm{~nm}$ wavelength that was used in this project. Thanks to these features it is relatively easy to couple light coming out of the other side of the fibre. Chip is enclosed in translucent DIP package and one must only avoid to illuminate those parts which are not photodiode.

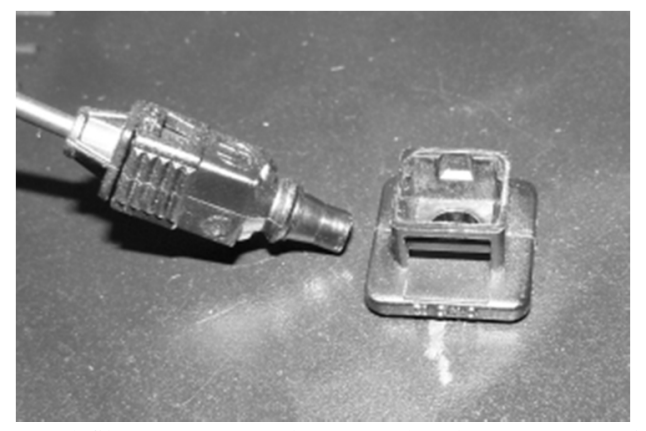

Fig. 4. Re-used fibre connectors that proved to be very convenient when light from fibre needs to be coupled onto DIP8 package (not shown, below socket). 
Strong light directed towards non sensitive parts of the chip might alter its behaviour. If one thinks of some protection against such situation then a small bush or snap fit epoxied to chip should help. OVKD02 female socket made by Hirschmann (now Belden subsidiary) was reused here. It proved to be very handy as it quite accurately snap fits DIP8 package of OPT101 from above. Therefore light is lead in a way that it affects only proper area of chip due to small distance between end of the fibre and OPT101 photodiode. OVKD02 socket embedded in transducer case and OVKS fibre male connector are shown on Fig. 4. Photodiode, socket and fibre plug still can be easily disassembled for maintenance and transport.

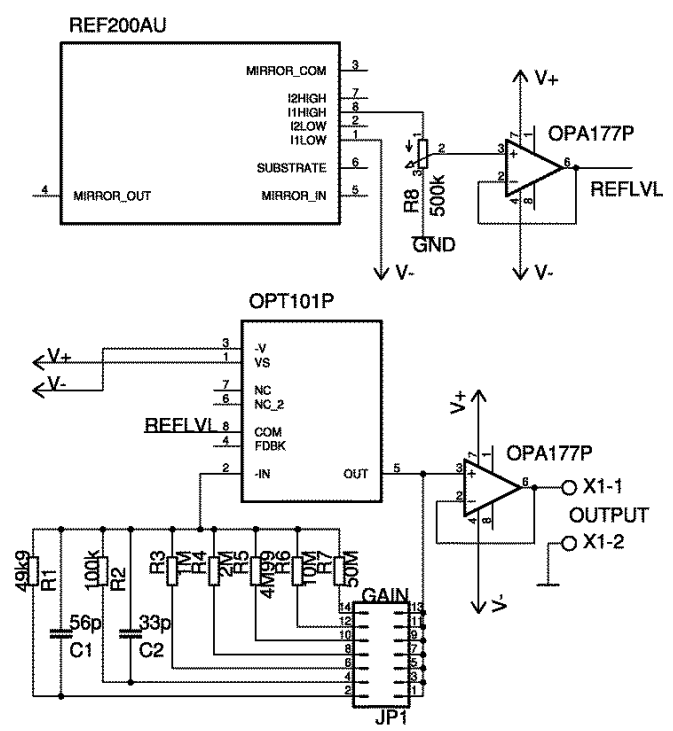

Fig. 5. Circuit diagram of optoelectronic transducer for turbidity measurements. Decoupling capacitors removed to make the figure neat.

Electronic circuit created for this project follows guide presented in OPT101 datasheet. Its diagram is shown on Fig. 5. Bipolar 3,3 V supply was used to match it with XBee board.

In turbidimeters an ambient light might cause a problem as it introduces some undesirable base offset. Fighting stray and ambient light which can enter the enclosure would be pointless as the same light might get into the fibre and find its path to photodiode anyway. Therefore an adjustment circuit is needed so it is possible to counteract this "dark error". With OPT101 it is possible to change reference voltage at photodiode anode which is accessible at pin 8 . It is supplied through OPA177 that is fed by potentiometer voltage divider. Position of a wiper can be changed with dial that is installed on the front panel so user can calibrate output voltage to $0 \mathrm{~V}$ in case when sample is pure, deionized water.

OPT101 output is available through unity gain operational amplifier working as a buffer. It is connected to BNC connector placed in front panel and also to 10-bit ADC input of the Xbee which is an inexpensive wireless interface based on IEEE 802.15.4 standard. Data is collected remotely and transmitted to PC computer to which a similar XBee board is connected. Programs to collect and process received data were written in Python language.

User can choose one of seven gains with a knob switch mounted on front panel. Two lowest gains that are fractional gains of 0,05 and 0,1 turned out to be the most useful for turbidity values in tested range that was up to $800 \mathrm{NTU}$ (Nephelometric Turbidity Unit). Different and less expensive laser with lower power could be used on higher gains and for smaller turbidities. To increment board supply is another choice but then transducer output exceeds sampling range of the XBee.

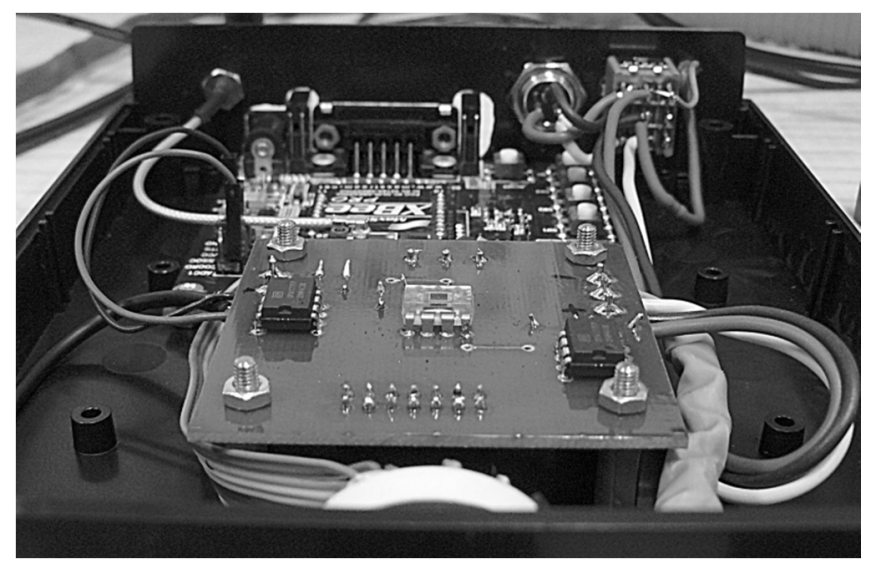

Fig. 6. Prototype version of the device with top lid removed. Transducer board is in the front with OPT101 visible in the middle. XBee board is at the back.

Board was designed with popular and cost-free version of CadSoft Eagle. After manual soldering it was arranged together with XBee board in single enclosure. Final prototype of the assembled device is presented on Fig. 6.

\section{RESUlTS}

Finally a laboratory stand was organised when optical system on breadboard and transducer were both operative. Supply voltages were measured with Escort 97 multimeters. Output from transducer was occasionally observed with DSO-X 2022A oscilloscope and measured with HP34401A laboratory multimeter. All further measurements of turbidity and discussion refer to and are based on an arrangement that is shown on Fig. 7.

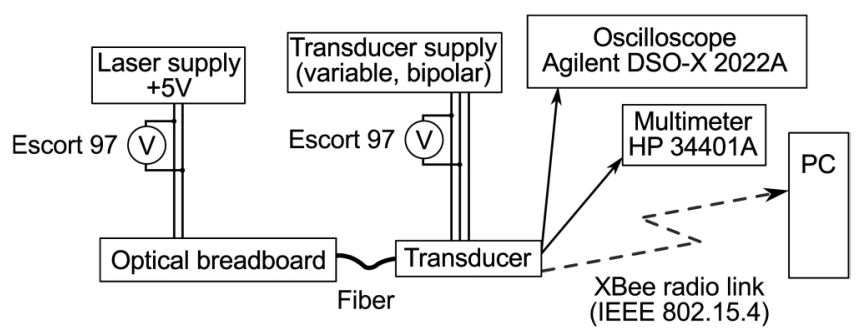

Fig. 7. Arrangement of laboratory stand.

In preliminary test it turned out that with used laser for low turbidities, such as of tap water, the smallest available, fractional gain of $50 \mathrm{kV} / \mathrm{A}$ is sufficient. It is achieved with feedback $50 \mathrm{k}$ resistor in parallel with $56 \mathrm{pF}$ capacitor. With bipolar $3,3 \mathrm{~V}$ supply voltage $(6,6 \mathrm{~V}$ rail-to-rail) maximum output voltage is about $+2,5 \mathrm{~V}$ mostly due to $0,7 \mathrm{~V}$ drop. Results from simple calculation show that current equals $0,05 \mathrm{~mA}$. OPT101 responsivity is $0,45 \mathrm{~A} / \mathrm{W}$ at $650 \mathrm{~nm}$ according to the datasheet so theoretical maximum measured radiant power equals $0,1(1) \mathrm{mW}$. Experiment results fit characteristics presented in datasheet thus might be considered as a confirmation that device 
works properly.

Base materials for further experiments were 200, 500 and 800 NTU water suspensions of formazine that are traceable to Polish national reference standard. All were ordered from professional chemical laboratory that prepared them according to PN-EN ISO 7027 norm [10]. These base solutions were further diluted by pipetting with pure water to create series of samples with lower turbidity values spanning from 5 NTU up to 800 NTU. Measurements were performed on two ranges - with gains 0,05 and 0,1 . Every point consists of 80 measurements that were done every second and then averaged.

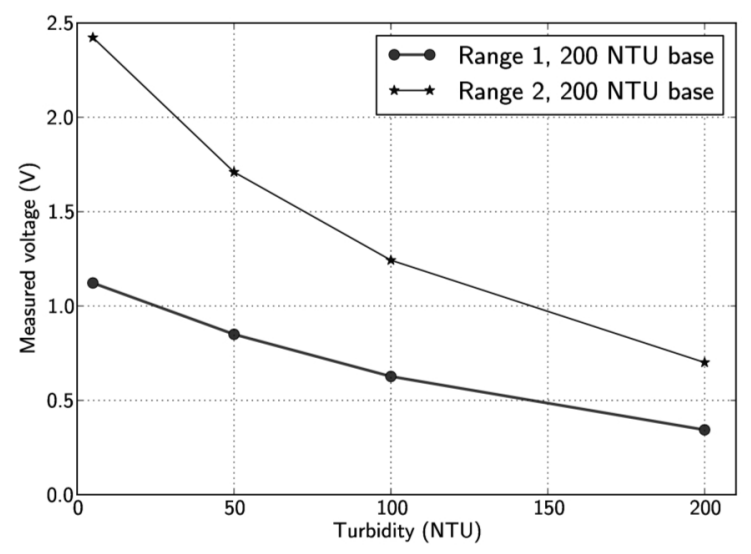

Fig. 8. Measurements of lower turbidity samples.

In the first experiment low turbidities that are typical for natural waters were measured. Tap water should have less than 5 NTU while clean lake is usually less than 100 NTU. Preparing standard samples with so low values is tricky. Created samples of 5, 50 and 100 NTU were based on 200 NTU standard suspension. Laser light had to be attenuated with polariser so for sample with the lowest NTU the voltage response of the transducer was within ADC sampling range. This characteristic is shown on Fig. 8.

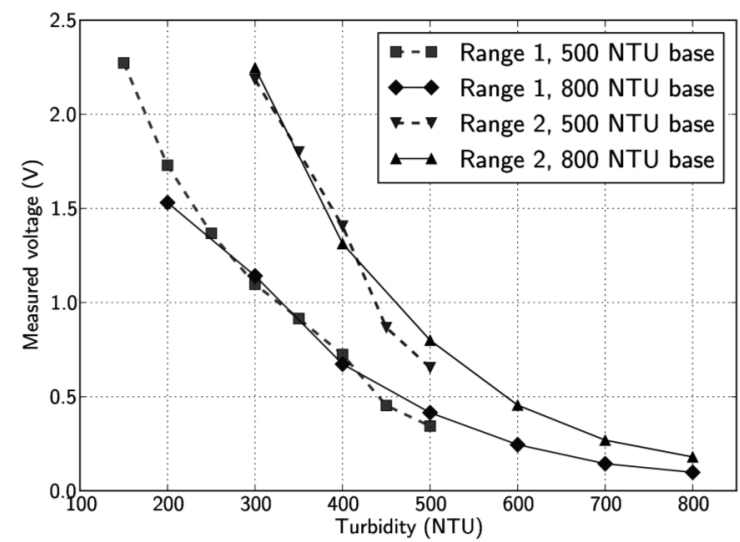

Fig. 9. Measurements of higher turbidity samples.

Two series of higher turbidities that are typical for natural, inland reservoirs were measured with more irradiant power (i.e. polariser was "opened"). Second series was from 150 to $500 \mathrm{NTU}$ with step of $50 \mathrm{NTU}$ while third was in range from 300 to 800 NTU with 100 NTU step. These two series, each measured on two ranges are shown on Fig. 9.
Series correlate to each other in the subset where they overlap at both of used ranges. It means that dilution processes, which started at different points, were done properly.

It is visible that one range has twice the gain of the other as anticipated. Shape of curves confirms that device works according to Lambert-Beer law. Gathered data might be foundation for calibration procedure in future experiments.

\section{CONCLUSIONS}

In this article it was shown how an integrated circuit of amplified photodiode might speed up development of turbidimeter. An inexpensive transducer which is also easy to reproduce is result of this research. Main advantage of the device is its quite linear characteristic in range up to 200 NTU. This range is especially interesting as such turbidity values represent water that can be perceived as clean but in fact still requires filtration before consumption.

Some issues were found in the prototype of the transducer circuit. However these concerns are relatively negligible compared to overall good performance and significant capabilities of the stand. Probe that can be immersed or embedded in water pipes is to be developed on this basis.

Development of smaller, cheaper and easier to use turbidimeters will continue. This progress might bring wider adoption of such equipment in ordinary households. It should be especially beneficial for rural areas where water system is usually more hazardous due to distance from water treatment plant or due to dubious local sources. Making devices that are also capable of wireless transmission will make them simpler to connect with modern environments of "smart homes" and "smart cities".

\section{REFERENCES}

[1] Council Directive 98/83/EC of 3 November 1998 on the quality of water intended for human consumption. [Online]. Available: http://eur-lex.europa.eu/legal-content/EN/TXT/?uri=CELEX: 31998L0083

[2] I.R. Kenyon, the Light Fantastic. Oxford University Press, 2008.

[3] W. Hergert, T. Wriedt, "The Mie theory. Basics and applications", Springer Series in Optical Sciences, vol. 169, 2012.

[4] J. Mroczka, D. Szczuczynski, "Improved technique of retrieving particle size distribution from angular scattering measurements", Journal of Quantitative Spectroscopy and Radiative Transfer, vol. 129, 2013.

[5] M. Jonasz, G. R. Fournier, Light Scattering by Particles in Water. Theoretical and Experimental Foundations. Elsevier, 2007.

[6] M. Sadar, Introduction to Laser Nephelometry, an Alternative to Conventional Particulate Analysis Methods. Hach Company, 2005.

[7] R. Varma, H. Moosmuller, W. P. Arnott, "Toward an ideal integrating nephelometer", Optics Letters, vol. 28, no. 12, 2003, pp. 1007-1009. [Online]. Available: http://dx.doi.org/10.1364/OL.28.001007

[8] A. Michalski, B. Dziadak, L. Makowski, "Some practical problems of communications reliability in environmental monitoring systems", Metrology and Measurement Systems, vol. 20, no. 3, pp. 337-350, 2013.

[9] B. O'Flyrm, R. Martinez, J. Cleary, C. Slater, F. Regan, D. Diamond, H. Murphy, "SmartCoast: A wireless sensor network for water quality monitoring", 32nd IEEE Conf. on Local Computer Networks, (LCN 2007), 2007, pp. 815-816.

[10] "ISO 7027: Water quality -- Determination of turbidity". International Organization for Standardization. Geneva, Switzerland. 1999. [Online]. Available: http://www.iso.org/iso/catalogue_detail? csnumber $=30123$ 